\title{
Giving voice to Black women in science and medicine
}

\section{To the Editor - Susan Moore was a} well-regarded Black physician who contracted SARS-CoV-2 in 2020. During her hospitalization, Dr. Moore released a public video detailing concerns that she was receiving inequitable treatment for COVID-19, having her complaints of pain dismissed, and experiencing medical laxity from her hospital team ${ }^{1}$. Unfortunately, she was discharged home and eventually returned to a different emergency room 12 hours later, where she died from COVID-19. The pain mismanagement that Dr. Moore experienced is an example of the glaring treatment disparity experienced by Black patients ${ }^{2}$. Dr. Moore's tragic death raised a heart-wrenching and disappointing thought among the authors of this Correspondence, all of whom are Black biomedical scientists and/or clinicians. If we cannot defend our own Black bodies, then what power do we truly have to push back against bias while defending our patients? The feeling that we are putting our lives on the line to still die disproportionately is a common sentiment among Black clinicians and trainees ${ }^{1}$. Dr. Moore's story is a horrific reminder that despite our ability and efforts as clinicians and scientists to advocate for equitable healthcare, protection for our own Black bodies against unconscious bias, ignorance and racial bigotry is not guaranteed.

While the United States grappling with its systemic racism is framing the current social climate, the COVID-19 pandemic is accentuating racial disparities in healthcare. Therefore, it is imperative for the biomedical community to recognize the vulnerable position of Black women in medicine ${ }^{3}$. Chronic exposure to racism and unfair treatment contribute to physiological and psychological deterioration ${ }^{4}$, and Black women in science and healthcare must confront racism and sexism in society and at work. To bring visibility to the tangible impact that health inequity has on Black women in science and medicine, Eseosa Ighodaro (a physician-scientist and first author of this Correspondence) organized an online, public, expert panel discussion entitled 'Tragedy: The Story of Dr. Susan Moore and Black Health Disparities' (https://youtu.be/dkGkLVgDfnw) ${ }^{2}$. Panelists and audience members shared experiences unique to Black female clinicians and scientists from across a diverse span of career stages. This online event created opportunities for community building and social support among Black clinicians and scientists. Many participants shared stories about a family member or friend who fell victim to COVID-19 and health inequity.

With cultural competency training, a healthcare provider could have been able to consider the role of bias in pain management and respond appropriately to Dr. Moore's symptoms ${ }^{5,6}$. Incorporating effective cultural and health-disparity competency training throughout all levels of healthcare training is essential. The training should include contextualization of the social determinants of health, along with knowledge of systemic factors that drive health disparities ${ }^{5,7}$. This would expand clinicians' understanding of medicine as inextricably operating within a socio-ecological framework shaped by structural racism that directly impacts health outcomes.

Resources to protect Black trainees, physicians and scientists from everyday discrimination and structural racism must be embedded into training programs, as well as the workforce infrastructure of academic medical institutions. Working in an environment in which Black people feel they are experiencing unfair treatment and frequent discrimination can take a toll on their well-being and psychological health, as reported by medical trainees ${ }^{3,8}$. Our allostatic load, a multisystem index of biological dysregulation, is disproportionately greater than that of our white counterparts, in response to discrimination ${ }^{4}$. Interventions that address the specific burden of stress and anxiety experienced by under-represented healthcare providers and scientists, such as mindfulness and social support, could improve the wellness ${ }^{4,9}$ and retention of under-represented trainees. A deepened commitment to the inclusion of people of color in the field of medicine alone helps decrease health disparity ${ }^{10}$. The US National Institutes of Health has created an initiative, the Faculty Institutional Recruitment for Sustainable Transformation program, that is designed to create cultures of inclusive excellence at institutions funded by the US National Institutes of Health. This initiative is an example of a career-development program with a goal of getting academic institutions to hire cohorts of diverse people to decrease the attrition of Black professionals in academic medicine.

We understand that the solutions to eliminate health disparities and create an equitable workplace in biomedical sciences and healthcare are complex. Addressing barriers to equity at any single level of influence will not be enough fully to diminish health disparities. All stakeholders with an interest in advancing medicine and biomedical research must commit to prioritizing health equity. The medical community must also acknowledge that until Black professionals are represented at every level of decision-making in healthcare, biomedical research and medical education, the power to save every future Dr. Susan Moore lies just beyond reach. We will continue to say her name and shed light on racial inequalities in medicine.

Eseosa T. Ighodaro (D1,4凶, Erica L. Littlejohn ${ }^{2,4}$,
Adesuwa I. Akhetuamhen (D ${ }^{3}$ and
Richard Benson $\mathbb{D}^{2}$
${ }^{1}$ Department of Neurology, Mayo Clinic,
Rochester, MN, USA. ${ }^{2}$ Office of Global Health
and Health Disparities, National Institute of
Neurological Disorders and Stroke, National
Institutes of Health, Bethesda, MD, USA.
${ }^{3}$ Department of Emergency Medicine, McGaw
Medical Center of Northwestern University,
Chicago, IL, USA. ${ }^{4}$ These authors contributed equally:
Eseosa T. Ighodaro, Erica L. Littlejohn.
${ }_{\text {e-mail: ighodaro.eseosa@mayo.edu }}$

Published online: 12 July 2021

https://doi.org/10.1038/s41591-021-01438-y

References

1. Givens, R. N. Engl. J. Med. 384, e18 (2021).

2. Ighodaro, E. T. \& Spector, A. Nature 591, 369 (2021)

3. Osseo-Asare, A. et al. JAMA Netw. Open 1, e182723 (2018).

4. Ong, A. D. et al. Cultur. Divers. Ethnic Minor. Psychol. 23, 27-35 (2017).

5. Metzl, J. M. \& Hansen, H. Soc. Sci. Med. 103, 126-133 (2014).

6. Jongen, C. et al. BMC Health Serv. Res. 18, 232 (2018).

7. Sharma, M. et al. Acad. Med. 93, 25-30 (2018)

8. Perry, S. P. et al. J. Racial Ethn. Health Disparities 3, 519-526 (2016).

9. Conway-Phillips, R. et al. Complement. Ther. Med. 48, 102277 (2020).

10. Greenwood, B. N. et al. Proc. Natl Acad. Sci. USA 117, 21194-21200 (2020). 
omitted. This Correspondence is signed in an individual capacity. The views and opinions expressed do not necessarily reflect that of any organization we (the authors) are associated with or employed by. This Correspondence does not represent the official view of the National Institute of Neurological Disorders and Stroke, the US National Institutes of Health, or any part of the US federal government. No official support or endorsement of this article by the National Institute of Neurological Disorders and Stroke or the US National Institutes of Health is intended or should be inferred. All authors have read the Nature Research Authorship Policy and confirm that this Correspondence complies.
Author contributions

E.L.L., E.T.I. and A.I.A conceived of and drafted the manuscript; and all authors edited and revised the manuscript and approved the final version of the manuscript.

\section{Accelerating the translational medicine cycle: the Academia Europaea pilot}

To the Editor-Translational Medicine (TM) is among the main challenges of the 21 st century; its development is essential for the application of scientific results for community benefit.

Although several developments have been achieved in the field, there is still room for improvement in implementing scientific results in healthcare. More than 1.4 million articles are published each year, as reported on PubMed; however, much of this knowledge is not applied in everyday practice. Based on a report from the European Commission's European Statistical Office, around 1.7 million people under 75 years of age die in Europe every year, but 1.2 million of these deaths could have been avoided through effective primary prevention and public-health intervention ${ }^{1}$.

The Academia Europaea, one of the leading advisory bodies of the European Commission, thus initiated the development of a new TM model that facilitates and accelerates the application of scientific knowledge for community benefit ${ }^{2}$. The new cycle model equally focuses on healthcare, the acquisition of new scientific findings, the digestible summation of results, and the communication of scientific knowledge to all stakeholders, including patients, healthcare professionals, pharmaceutical companies and policymakers. The model has been piloted at the Centre for Translational Medicine at the University of Pécs (UP-CTM) in Hungary, with great success, which made UP-CTM one of the most efficient and visible centers for TM in Eastern and Central Europe within five years.

The TM cycle model in healthcare was trialed in an eight-bed UP-CTM patient care unit that focused on managing acute pancreatitis. Patient coordinators, clinical research administrators, $\mathrm{PhD}$ students, nurses, and junior and senior doctors joined forces in the day-to-day work. This team ensured the application of available evidence-based guidelines and, notably, built up activities for healthcare delivery science-for example, the team enrolled patients in clinical trials and patient registries, and organized and analyzed the diagnostic and treatment pathways. In a year, we achieved a two-day reduction in the length of hospitalization per patient, a marked decrease in antibiotics administration, and a two-thirds drop in mortality. Due to the healthcare-delivery-science activity, patient-care costs also fell by $25 \%$ (ref. ${ }^{3}$ ) (Fig. 1a).

The model was subsequently tested in two other hospitals. At the Szent György University Teaching Hospital of Fejér County (Székesfehérvár, Hungary), a similar healthcare delivery science-based patient care unit for acute pancreatitis was launched. As a result, the use of antibiotics substantially decreased. The duration of hospitalization dropped by an average of one day per patient (Fig. 1b). At the Heim Pál National Pediatric Institute (Budapest, Hungary), a scientifically constructed cystic-fibrosis registry, which also examined glucose metabolism in detail, facilitated the early diagnosis of diabetes mellitus in $14 \%$ of the patients; these would have been missed by the previous system. In addition, nine children were identified as having impaired glucose tolerance; this may allow earlier diagnosis and treatment of established cystic fibrosis-related diabetes.

After the projects involving acute pancreatitis, we broadened our patient registries and clinical trials to a wide range of medical disciplines (Fig. 1c,d). Notably, these clinical databases and biobank samples substantially added to the 300 publications by UP-CTM over the five years, which may substantially contribute to the implementation of scientific results for community benefit. The outstanding networking is well illustrated through UP-CTM's joint publications involving more than a thousand researchers in the five years (Fig. 1e).

To support patient care, scientific activity and communication, we set up an interdisciplinary unit at the outset with several different disciplines, including patient coordination, biostatistics, informatics, data management, artificial intelligence, legal support and communication. Notably, the unit accelerated scientific activity, supported patient care and communication, and created 51 jobs with new professional content by the end of the five years, which is extremely important in a century during which the demand for professionals is decreasing in numerous fields (Fig. 1f).

A shift was necessary for the academic field and communication as well. Medical professionals must understand scientific results to deliver high-quality patient care; therefore, our center developed a new 'learning by doing' education module in which 45 young physicians learned the scientific methodology. The program's strength is emphasized by the fact that doctors from ten different cities in four countries participated in the training program. At the same time, we developed a complex Clinical Research Administrator training program accredited and tested in the fifth year. During this period, printed educational materials and online educational videos were made available for healthcare professionals; these have been particularly useful during the COVID-19 pandemic. Grant committees also recognized the potential of the TM cycle approach (Fig. 1g). 